\title{
Distribuição de Heteroptera Aquáticos (Insecta) em Diferentes Tipos de Substratos de Córregos do Cerrado Matogrossense
}

\author{
Karina Dias-Silva ${ }^{\circledR}$, Helena Soares Ramos Cabette² ${ }^{2}$, Núbia França da Silva Giehl² \& Leandro Juen³
}

1. Universidade Federal de Goiás, e-mail: karinabionx@gmail.com (Autor para correspondência ${ }^{\bowtie}$ ). 2. Universidade do Estado de Mato Grosso, e-mail: hcabette@uol.com.br, nubiagiehl@gmail.com. 3. Universidade Federal do Pará, e-mail: leandrojuen@ufpa.br

EntomoBrasilis 6(2): 132-140(2013)

\begin{abstract}
Resumo. A comunidade de Heteroptera de córregos do cerrado do Leste Matogrossense foi analisada para determinar os tipos de substratos que contemplam a maior variação de composição de espécies. Foram realizadas coletas nos substratos de folhiço de corredeira e remanso, raiz, cascalho, pedra (matacão), areia e na superfície d'água (camada de tensão superficial) das nascentes, trechos médios e foz dos córregos Santo Antônio e Colher em dezembro/2004 e julho/2005, com cada substrato representado por seis subamostras. Foram coletados 333 indivíduos distribuídos em 11 famílias, 20 gêneros e 30 morfoespécies (17 Nepomorpha e 13 Gerromorpha), sendo os Gerromorpha mais abundantes (172 indivíduos). A riqueza estimada de Gerromorpha nos substratos foi superior em coletas na superfície d'água, enquanto Nepomorpha apresentou maior riqueza em raiz, seguido por cascalho e folhiço de corredeira. Os substratos inorgânicos (pedra e areia) apresentaram reduzida riqueza de Heteroptera. Entre os Gerromorpha, Limnogonus aduncus aduncus Drake \& Harris, Rhagovelia elegans Uhler, Neogerris lubricus White e Brachymetra sp.1 foram associados com coletas de superfície e Stridulivelia anta Polhemus \& Spangler com substrato raiz. Em Nepomorpha, Martarega chinai Hynes foi associado com coletas de superfície, enquanto que Ambrysus sp. 1 mostrou associação ao folhiço de corredeira, de remanso, raiz e superfície. Com esses resultados ressaltamos que trabalhos que visem levantamentos rápidos da comunidade de Heteroptera devem priorizar coletas de superfície para Gerromorpha e de raiz para Nepomorpha, pois esses substratos abrigam as maiores riquezas de cada infraordem, contemplando $83 \%$ e $64 \%$ da riqueza de espécie desses grupos respectivamente.
\end{abstract}

Palavras-chave: Insetos Aquáticos; Microhabitat; Riachos.

\section{Distribution of Aquatic Heteroptera (Insecta) From Different Types of Streams Substrates of Cerrado in Mato Grosso}

Abstract. The Heteroptera community of Cerrado streams in east of Mato Grosso was analyzed to determine the types of substrate that contemplate the largest variation in species composition. Samples were performed in the leaf litter substrates from rapids and backwaters, root, gravel, stone (boulder), sand and on the water surface (surface tension layer) of springs, mean stretches and mouths of the Santo Antônio and Colher streams on December/2004 and July/2005, with six subsamples each substrate. Were collected 333 specimens distributed in 11 families, 20 genus and 30 morphospecies (17 Nepomorpha and 13 Gerromorpha), being Gerromorpha the most abundant (172 specimens). The estimated richness of Gerromorpha in the substrates was higher in samples from water surface, while Nepomorpha showed higher richness in root, followed by gravel and leaf litter from rapids. The inorganic substrates (stone and sand) showed lower richness of Heteroptera. Among Gerromorpha, Limnogonus aduncus aduncus Drake \& Harris, Rhagovelia elegans Uhler, Neogerris lubricus White and Brachymetra sp.1 were associated to surface samples and Stridulivelia anta Polhemus \& Spangler to root substrate. In Nepomorpha, Martarega chinai Hynes was associated to surface samples, while Ambrysus sp. 1 showed association to leaf litter from rapids, backwaters, root and surface. The results suggest that studies which aim for rapid surveys of Heteroptera community should prioritize surface samples for Gerromorpha and root samples for Nepomorpha, because these substrates shelter the greatest richness of each infra-order, contemplating $83 \%$ and $64 \%$ of species richness in these groups respectively.

Keywords: Aquatic insects; Microhabitat; Streams.

(0) s insetos aquáticos têm uma estreita relação com o tipo de substrato onde podem ser encontrados em riachos (HyNes 1970; KiKuChi \& UiEdA 2005), uma vez que estes criam condições de habitat específicas (Buss et al. 2003). Essas condições propiciam diferentes formas de alimentação e abrigo contra correnteza e predadores, sendo, um dos principais fatores que afetam sua distribuição e abundância (WISE \& MoLles 1979; RiBEIro et al. 1998). Consequentemente, o conhecimento sobre a diversidade de habitat e a relação destes com a fauna é uma importante ferramenta para a avaliação das condições ambientais dos ecossistemas aquáticos (GALDEAN et al. 2000).

Heteroptera é um grupo predador, encontrado em todos os ambientes (Neri et al. 2005), tendo indivíduos aquáticos representados por Nepomorpha e semi-aquáticos representados por Gerromorpha (NIESER \& Melo 1997). Esses organismos vivem em remansos de rios, riachos, lagos e pântanos (RoLDÁN-PÉREZ 1988) e apresentam baixa resistência a correntezas rápidas. Alguns grupos como Naucoridae, Notonectidae e Belostomatidae, desempenham o papel de controladores biológicos de larvas e pupas de mosquitos vetores de doenças (Menke 1979; Petts 1994).

Segundo Dias-Silva et al. (2010) são conhecidas 67 morfoespécies de Heteroptera para a região leste do Mato Grosso. Estes insetos apresentam distribuição agregada nos ambientes aquáticos, sofrendo influência direta ou indireta da profundidade, 
T correnteza, tipo de substrato, disponibilidade e qualidade de detritos orgânicos e de alimentos, de modo que a oferta de substratos, ocorrência de longas estiagens, influência antrópica e escassez de alimento podem alterar a distribuição das espécies (CAllisto et al. 2002).

Em Heteroptera, o esperado é que os Gerromorpha, que vivem e perseguem suas presas deslizando sobre a película superficial da d'água (Schuh \& Slater 1995; HeCKMAN 2011), sejam encontrados com maior frequência na superfície do que nos outros substratos; por outro lado, os Nepomorpha, por serem bentônicos devem apresentar relações mais estreitas com substratos de fundo (e.g. Naucoridae), assim como os nectônicos, que buscam nesses substratos abrigo e presas (e.g. Corixidae) (Hungerford 1948).

O objetivo do estudo foi avaliar a preferência de Heteroptera aquáticos e semi-aquáticos por tipo de substrato em córregos do cerrado matogrossense. As hipóteses são: (i) Nepomorpha (maioria de seus representantes apresentam modo de vida bentônico) apresentarão maior riqueza em substratos orgânicos (folhiço e raiz), uma vez que estes substratos podem oferecer maior disponibilidade de presas e abrigo contra predadores, (ii) Gerromorpha, devido seu modo de vida de superfície, apresentará maior riqueza na lâmina d'água.

\section{MATERIAL E MÉTODOS}

Área de estudo. Os Córregos Santo Antônio e da Colher (Figura 1) estão situados à margem esquerda do médio Rio das Mortes, leste do Mato Grosso, sendo o clima da região caracterizado como Tropical Continental alternadamente úmido (tipo II, subtipo c), com temperatura média anual variando de 22,2 a $23,4^{\circ} \mathrm{C}$ e precipitação de 1500 a 1800 mm (Mato Grosso 1998). Esses riachos são considerados de $3^{\mathrm{a}}$ ordem, STRAHLER em PETTS (1994) e sofreram alterações pela influência da prática de garimpo de ouro, no período de 1981 a 1993, e posteriormente pelas práticas de pecuária extensiva com a introdução de gramíneas exóticas.

Em cada córrego foram selecionados três sítios amostrais denominados de nascente $\left(\mathrm{N}-1^{\mathrm{a}}\right.$ ordem), intermediário $\left(\mathrm{I}-2^{\mathrm{a}}\right.$ ordem) e foz ( $\mathrm{F}-3^{\mathrm{a}}$ ordem), respeitando uma distância mínima de três quilômetros entre eles (Tabela 1, características dos sítios amostrados).

Amostragens e análise de dados. As coletas foram realizadas no período chuvoso de dezembro/2004 e na estiagem de julho/2005. Em cada um dos sítios demarcados e período de coleta, foram amostrados cinco subamostras para os seguintes substratos: raízes submersas (R), areia (A), cascalho fino (C), folhiço de remanso (FR), folhiço de corredeira (FC), rochas grandes ou matacões ( $\mathrm{P}$, anotados aqui como pedras) e superfície da água (película de tensão superficial da água sobre a qual patinam os insetos) (S). Estes substratos foram escolhidos com base em Nieser \& Melo (1997) e Assis et al. (2004), por serem os tipos mais representativos para os insetos aquáticos, em conformidade com o ambiente estudado.

Para as coletas dos substratos areia, raiz e folhiços (remanso e corredeira), utilizamos coador manual com rede de $18 \mathrm{~cm}$ de diâmetro, $15 \mathrm{~cm}$ de profundidade e malha de $0,5 \mathrm{~mm}$. Para captura ativa na lâmina d'água o esforço de coleta foi de 10 minutos.

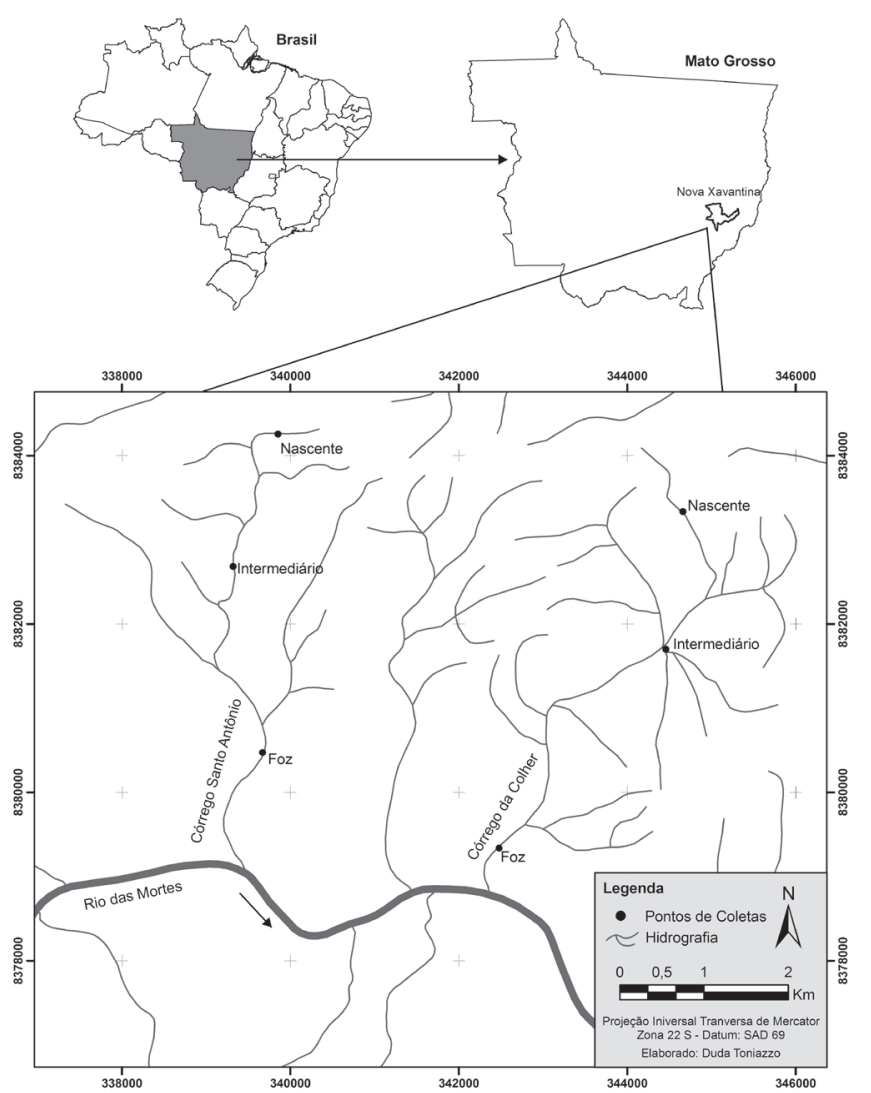

Figura 1. Localização dos pontos de coleta no Córrego Santo Antônio e Córrego da Colher, Nova Xavantina-MT.

Tabela 1. Caracterização dos locais de coleta, Nova Xavantina, MT, anotados em período de seca (jul. 2005). (Larg. - largura média; Prof.- profundidade média; veget. - vegetação; $1^{\mathrm{a}}$ a $3^{\mathrm{a}}$ - ordem do córrego).

\begin{tabular}{|c|c|c|c|c|c|c|c|c|}
\hline Local & $\begin{array}{r}\text { Coord } \\
\text { Sul e }\end{array}$ & $\begin{array}{l}\text { nadas } \\
\text { Deste }\end{array}$ & $\begin{array}{l}\text { Alt. } \\
\text { (m) }\end{array}$ & $\begin{array}{l}\text { Larg. } \\
\text { (m) }\end{array}$ & Prof. (m) & Substrato & Veget.ciliar & \multirow[t]{2}{*}{ Conservação } \\
\hline C. Sto. Antônio (CRSA) & $14^{\circ} 36^{\prime} 36,6^{\prime \prime}$ & $52^{\circ} 29^{\prime} 13,8^{\prime \prime}$ & 380,0 & & & & & \\
\hline Nascente $\left(1^{\mathrm{a}}\right)$ & & & & 2,43 & 0,18 & Areia e rochas & $\begin{array}{l}\text { Scheelea } \\
\text { (bacuri) }\end{array}$ & Regeneração \\
\hline Intermediário $\left(2^{\mathrm{a}}\right)$ & & & & 3,27 & 0,26 & $\begin{array}{l}\text { Blocos de rochas, } \\
\text { areia e cascalho }\end{array}$ & Mata de galeria & Preservado \\
\hline$F O Z\left(3^{\mathrm{a}}\right)$ & & & & 5,21 & 0,20 & $\begin{array}{l}\text { Matacões (rejeito } \\
\text { de garimpo) }\end{array}$ & Pastagem exótica & Alterado \\
\hline
\end{tabular}

Córrego Colher (CRCO) $\quad 14^{\circ} 37^{\prime} 8,6^{\prime \prime} \quad 52^{\circ}{ }^{\circ} 26^{\prime} 34,1^{\prime \prime} \quad 369,0$

Nascente $\left(1^{\mathrm{a}}\right)$

Intermediário $\left(2^{\mathrm{a}}\right)$
1,55

3,07

5,17

0,24

0,28

0,36

Argila e Areia
Areia e cascalho

Blocos de rochas e areia
Scheelea (bacuri)

Pastagem exótica e palmeiras

Mata de galeria sp. Regeneração

Alterado

A


Para cascalho removeu-se as rochas do fundo, e o material foi conduzido, pela corrente de água, para uma rede surber, que delimitava o espaço de coleta, para matacões, 15 rochas de cerca de $20 \mathrm{~cm}$ de diâmetro foram escovadas, sobre bandeja branca. Embora os métodos amostrais fossem diferentes, buscamos expressar volume/áreas amostrais similares.

O material foi triado em campo, preservado em álcool a $85 \%$ e identificado a partir das chaves de Nieser \& Melo (1997), NiEser et al. (1999) e Goodwyn (2001) até o nível de gênero e em seguida morfoespeciado e depositado na Coleção Zoobotânica "James Alexander Ratter", Universidade do Estado do Mato Grosso (UNEMAT)/Nova Xavantina (CZNX). Adultos das duas infraordens foram analisados separadamente devido a diferenças morfológicas e forma de utilização do ambiente aquático (NIESER \& MELO 1997).

A riqueza foi estimada com jackknife de $1^{\mathrm{a}}$ ordem utilizando o programa EstimateSWin 7.5.0 (COLWELL 2005), esse estimador utiliza um fator de correção para a riqueza observada dando um maior peso quando existem muitas espécies raras na comunidade (BURNHAM \& OvERTON 1978), para realizar a comparação da riqueza de espécies nos diferentes substratos utilizamos a técnica de inferência por intervalo de confiança, onde a riqueza observada entre os substratos só será diferente se a riqueza média de um não for sobreposta pelo intervalo de confiança do outro (GoTELLI \& ElLison 2011). Para testar a associação de cada morfoespécie pelo tipo de substrato utilizamos o índice de espécies indicadoras - INDVAL (Indicator value), esse índice mede a especificidade e a fidelidade das espécies a uma determinada condição ambiental previamente estabelecida, no caso do nosso estudo, tipos de substratos, o valor de significância $(\mathrm{p}<0,05)$ foi obtido através do teste de Monte Carlo usando 10.000 randomizações (DufrêNE \& LEGENDRE 1997).

\section{RESULTADOS}

Foram coletados 333 indivíduos distribuídos em 11 famílias, 20 gêneros e 30 espécies/morfoespécies. A infraordem Nepomorpha contribuiu com 161 e Gerromorpha com 172 indivíduos (Tabela 2). Ao comparar a riqueza de morfoespécies das duas infraordens, Nepomorpha apresentou uma riqueza estimada de 22,83 $\pm 2,77$ (média \pm intervalo de confiança), com oito morfoespécies a mais do que Gerromorpha $(14,93 \pm 1,65)$ (Figura 2). Dentre os Nepomorpha a família Naucoridae foi a mais abundante (Ambrysus, Limnocoris e Cryphocricos) com 118 indivíduos (73,3\% do total de Nepomorpha) e seis morfoespécies (MF), e em Gerromorpha foi a família Gerridae (Brachymetra, Cylindrostethus, Limnogonus e Neogerris) com 78 indivíduos (45,3\% do total de Gerromorpha) e cinco morfoespécies.

Nepomorpha apresentou maior riqueza primeiramente em raízes (R) $(16,83 \pm 1,54)$ e posteriormente na superfície da água $(9,50$ $\pm 1,71$ ), essa riqueza é significativamente diferente dos outros substratos. Para Gerromorpha, ocorreu o inverso, com S (14,17 $\pm 2,01)$ apresentando, em média, nove espécies a mais do que $\mathrm{R}$ $(5,67 \pm 1,05)$ (Figura 2).

Entre as famílias, Naucoridae (Nepomorpha) contribuiu com 118 dos 161 indivíduos coletados (Tabela 2), com o maior percentual de indivíduos em cascalho (C) (34\%), seguido de raízes (R) (21\%), folhiço de corredeira (FC) (19\%) e superfície da água (S) (10\%). Gerridae dentre os Gerromorpha, foi a família que mais contribuiu com número de indivíduos 78 dos 172 coletados, a maior presença foi em S (96\%).

$\mathrm{Na}$ análise de espécies indicadoras nos substratos (INDVAL, Tabela 3), sete espécies apresentaram associações significativas, e com base nesse resultado apresentamos graficamente a

Tabela 2. Abundância de morfoespécies de Heteroptera nos substratos amostrados nos Córregos Santo Antônio e Colher Nova Xavantina-MT, 2004/2005.

\begin{tabular}{|c|c|c|c|c|c|c|c|c|}
\hline \multirow{2}{*}{ Gêneros/morfoespécies } & \multicolumn{7}{|c|}{ Substratos } & \multirow{2}{*}{ Total } \\
\hline & $\mathbf{A}$ & $\mathbf{C}$ & FC & FR & $\mathbf{P}$ & $\mathbf{R}$ & $\mathbf{S}$ & \\
\hline
\end{tabular}

NEPOMORPHA

\section{Belostoma}

Belostoma sp.1

Belostoma sp.2 o o

o

o

$\begin{array}{llll}0 & 1 & 0 & 1 \\ 0 & 1 & 1 & 2\end{array}$

\section{Corixidae}

Tenagobia

Tenagobia sp.1

Gelastocoridae

Montandonius Melin

Montandonius sp.1

o

o

o

o

O

O

o

o

o

o

o

1

\section{Nerthra Say}

\section{Nerthra $\boldsymbol{c}$ f. buenoi Todd}

o

o

1

O

O

o

O

Naucoridae

Ambrysus

Ambrysus sp.1

Ambrysus teutonius La Rivers

o

o

O

Cryphocricos

Cryphocricos sp.1

0

$\begin{array}{ll}1 & 12 \\ 8 & 8\end{array}$

4

o

12
5

4

33

$4 \quad 29$

Limnocoris

Limnocoris machrisi Nieser \& Lopez-Ruf

o 18

$18 \quad 1$

$1 \quad 0$

O

1

10

o

20

Continua... 
Tabela 2. Continuação...

\begin{tabular}{|c|c|c|c|c|c|c|c|c|}
\hline \multirow{2}{*}{ Gêneros/morfoespécies } & \multicolumn{7}{|c|}{ Substratos } & \multirow{2}{*}{ Total } \\
\hline & $\mathbf{A}$ & $\mathbf{C}$ & FC & FR & $\mathbf{P}$ & $\mathbf{R}$ & $\mathbf{S}$ & \\
\hline Limnocoris pusillus Montandon & 1 & o & o & o & o & o & o & 1 \\
\hline Limnocoris minutus De Carlo & 8 & 12 & 3 & o & o & 6 & 4 & 33 \\
\hline \multicolumn{9}{|l|}{$\begin{array}{l}\text { Nepidae } \\
\text { Ranatra }\end{array}$} \\
\hline Ranatra sp.1 & o & o & o & o & o & 2 & o & 2 \\
\hline Ranatra sp.2 & o & o & o & 1 & o & 1 & 0 & 2 \\
\hline \multicolumn{9}{|l|}{$\begin{array}{l}\text { Notonectidae } \\
\text { Buenoa }\end{array}$} \\
\hline Buenoa sp.1 & o & o & o & o & o & o & 2 & 2 \\
\hline Buenoa sp.2 & o & o & o & o & o & 8 & o & 8 \\
\hline \multicolumn{9}{|l|}{ Martarega } \\
\hline Martarega chinai Hynes & 0 & o & o & 0 & o & 1 & 21 & 22 \\
\hline \multicolumn{9}{|l|}{$\begin{array}{l}\text { Ochteridae } \\
\text { Ochterus }\end{array}$} \\
\hline Ochterus sp.1 & 1 & o & o & o & o & o & o & 1 \\
\hline \multicolumn{9}{|l|}{ GERROMORPHA } \\
\hline \multicolumn{9}{|l|}{$\begin{array}{l}\text { Gerridae } \\
\text { Brachymetra }\end{array}$} \\
\hline Brachymetra sp.1 & o & 2 & o & o & o & o & 18 & 20 \\
\hline
\end{tabular}

\section{Cylindrostethus}

Cylindrostethus palmaris Drake \& Harris

o

o

o

o

o

o

9

9

\section{Limnogonus}

Limnogonus aduncus aduncus

Limnogonus sp.1

o

1

o

o

o

\section{Neogerris}

Neogerris lubricus White

o

o

o

o

o

o 14

\section{Hidrometridae}

\section{Hydrometra}

Hydrometra guianana Hungerford \& Evans

o $\quad$ o

rand

1

o

o

31

32

Mesoveliidae

\section{Mesovelia}

Mesovelia sp.1

o

o

o

0

$\begin{array}{lll} & & 1\end{array}$

\section{Veliidae \\ Paravelia}

Paravelia sp.1

\section{Rhagovelia}

Rhagovelia elegans

Rhagovelia sp.1

Rhagovelia sp.2

\section{Stridulivelia}

\begin{tabular}{lccccccc} 
Stridulivelia anta Polhemus \& Spangler & 0 & 0 & 0 & 1 & 0 & 50 & 4 \\
Stridulivelia sp.1 & 0 & 0 & 0 & 0 & 0 & 3 & 0 \\
\hline Total de indivíduos & $\mathbf{1 0}$ & $\mathbf{4 4}$ & $\mathbf{2 7}$ & $\mathbf{1 0}$ & $\mathbf{4}$ & $\mathbf{9 4}$ & $\mathbf{1 4 4}$ \\
Total de táxons & $\mathbf{3}$ & 7 & $\mathbf{6}$ & $\mathbf{5}$ & $\mathbf{2}$ & $\mathbf{1 5}$ & $\mathbf{1 8}$ \\
\hline
\end{tabular}


abundância dessas espécies para melhor visualização destas nos substratos (Figura 3 - Nepomorpha e Figura 4 - Gerromorpha). Para Nepomorpha houve associação de Martarega chinai Hynes com S (Figura 3A), ocorrendo quase que exclusivamente nesse substrato e Ambrysus sp.1, que se associou a vários tipos (R, FC, S, FR; Figura 3B). Gerromorpha apresentou associação com $\mathrm{S}$ para Cylindrostethus palmaris Drake \& Harris, Brachymetra sp.1, Neogerris lubricus White, Rhagovelia elegans Uhler e Limnogonus aduncus aduncus Drake \& Harris, enquanto que Stridulivelia anta Polhemus \& Spangler com R. Comparando a variação das médias de abundância e desvio padrão dessas espécies podemos verificar que as quatro morfoespécies apresentaram médias de ocorrência altas para S (Figura 4A-D) e em R para $S$. anta (Figura 4E).
Tabela 3. Preferência por substrato das espécies de Heteroptera apontada pelo IndVAL, amostradas nos Córregos Santo Antônio e da Colher, Nova Xavantina, MT (2004/2005) [FC- folhiço de corredeira, FR- folhiço de remanso- $\mathrm{R}$ - substrato raiz; $\mathrm{S}$ - superfície da água]

\begin{tabular}{lccc}
\hline \multicolumn{1}{c}{ Espécies } & (IV) & p & Associação \\
\hline Nepomorpha & & & \\
Ambrysus sp.1 & 0,79 & 0,001 & FC+FR+R+S \\
\hline Martarega chinai & 0,70 & 0,006 & $\mathrm{~S}$ \\
\hline Gerromorpha & & & \\
Brachymetra sp.1 & 0,77 & 0,002 & $\mathrm{~S}$ \\
Neogerris lubricus & 0,79 & 0,004 & $\mathrm{~S}$ \\
Rhagovelia elegans & 0,70 & 0,014 & $\mathrm{~S}$ \\
Limnogonus a. aduncus & 0.68 & 0,015 & $\mathrm{~S}$ \\
\hline Stridulivelia anta & 0,77 & 0,012 & $\mathrm{R}$ \\
\hline
\end{tabular}

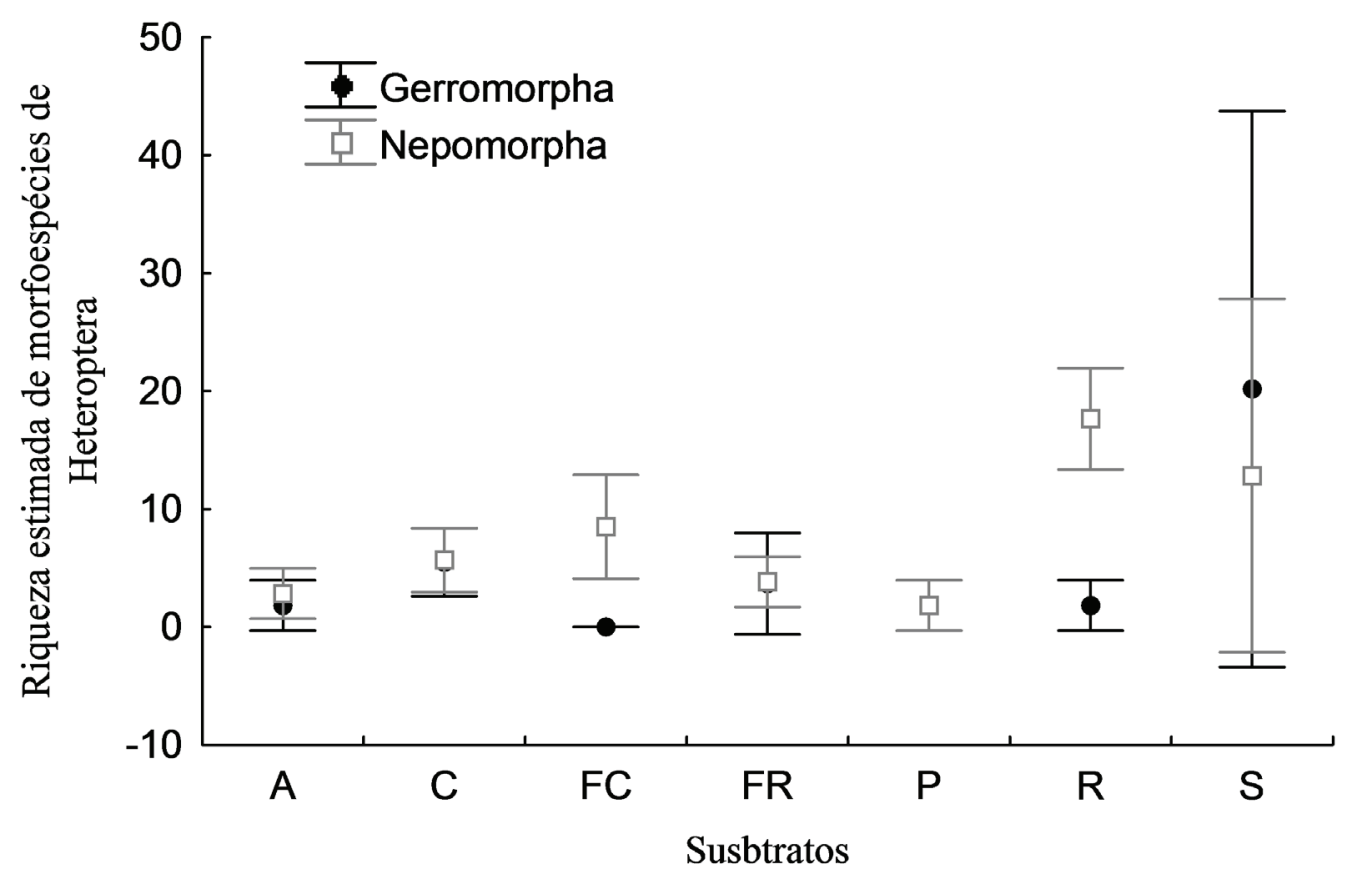

Figura 2. Riqueza de morfoespécies de Gerromorpha e Nepomorpha por substrato, estimada pelo procedimento jackkinife, dos córregos Santo Antônio e Colher, Nova Xavantina, MT (2004/2005) (as barras apresentam um intervalo de confiança de 95\%).
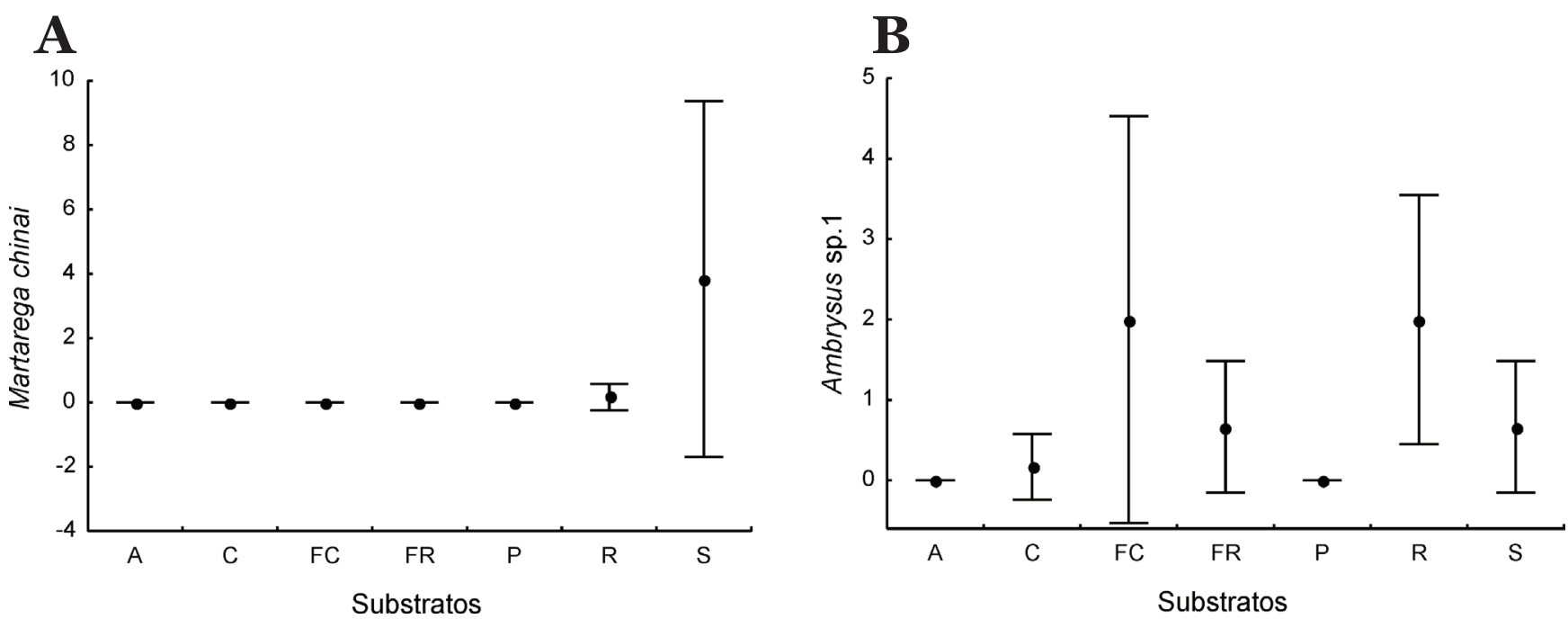

Figura 3. Abundância das morfoespécies de Nepomorpha nos substratos dos Córregos Santo Antônio e da Colher, Nova Xavantina, MT (2004/2005). A) Martarega chinai; B) Ambrysus sp.1 [A, areia; C, cascalho; FC, folhiço de corredeira; FR, folhiço de remanso; P, pedra; R, raiz e S, superfície]. 
A

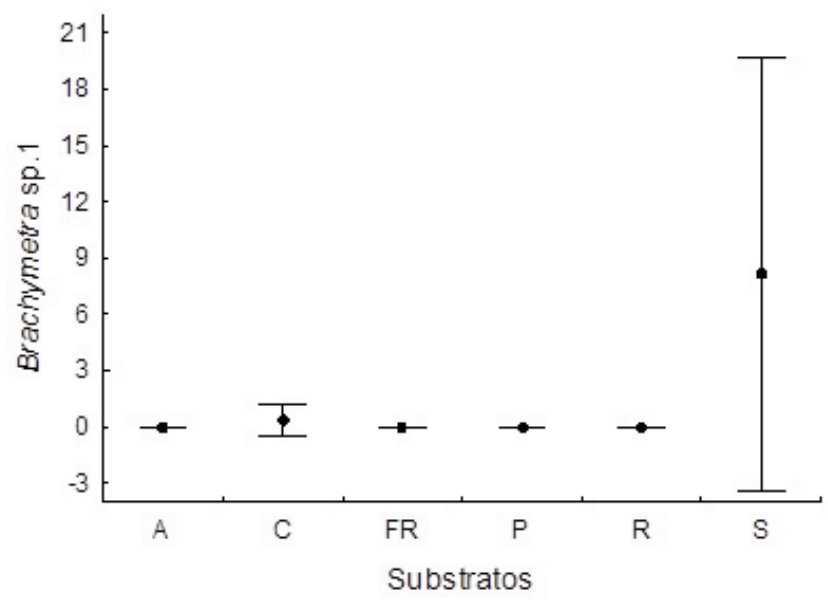

C

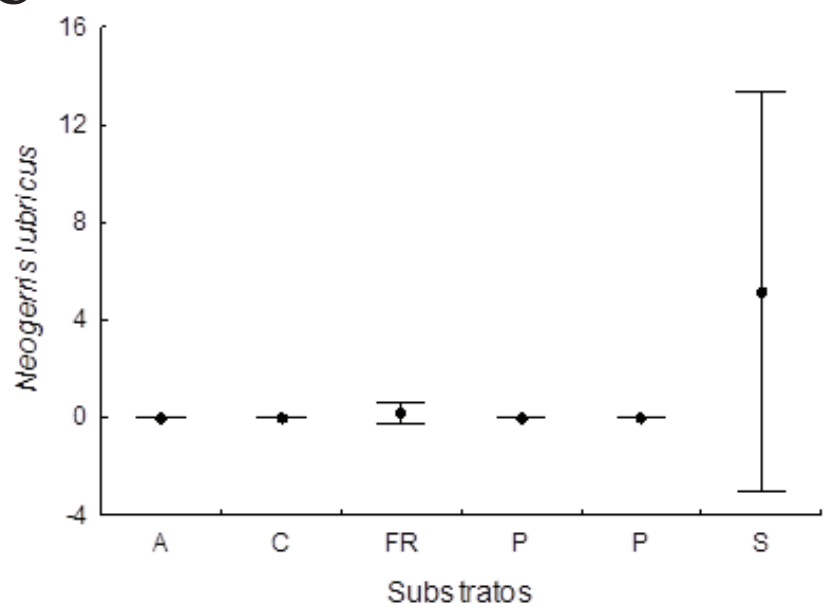

B

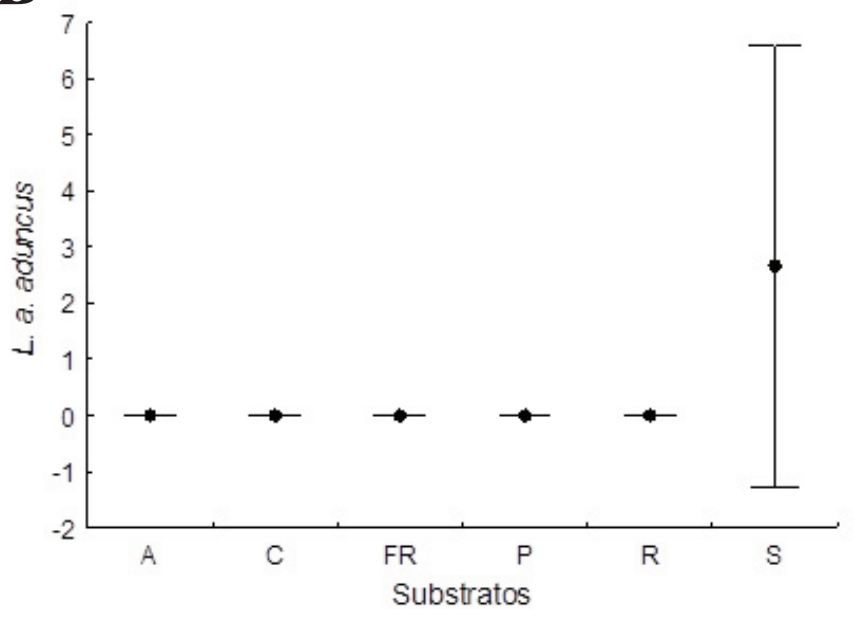

D

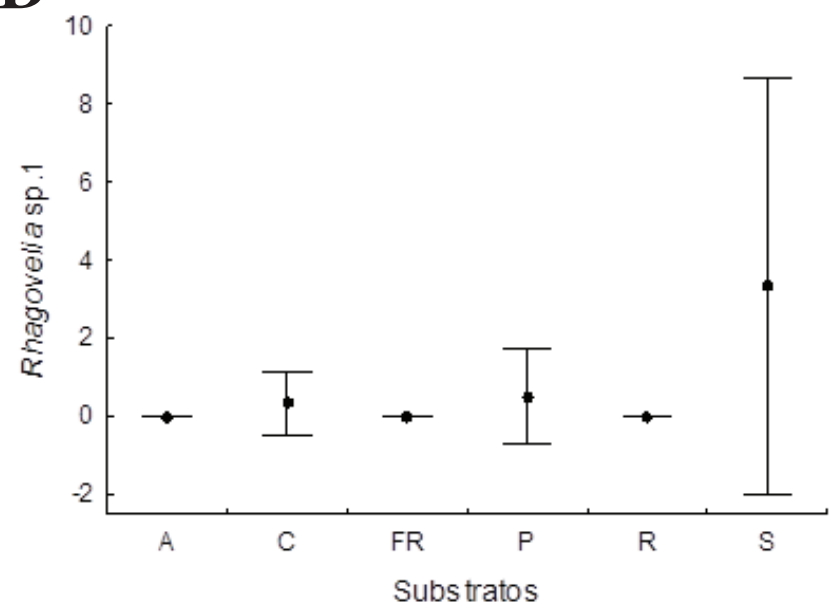

$\mathbf{E}$

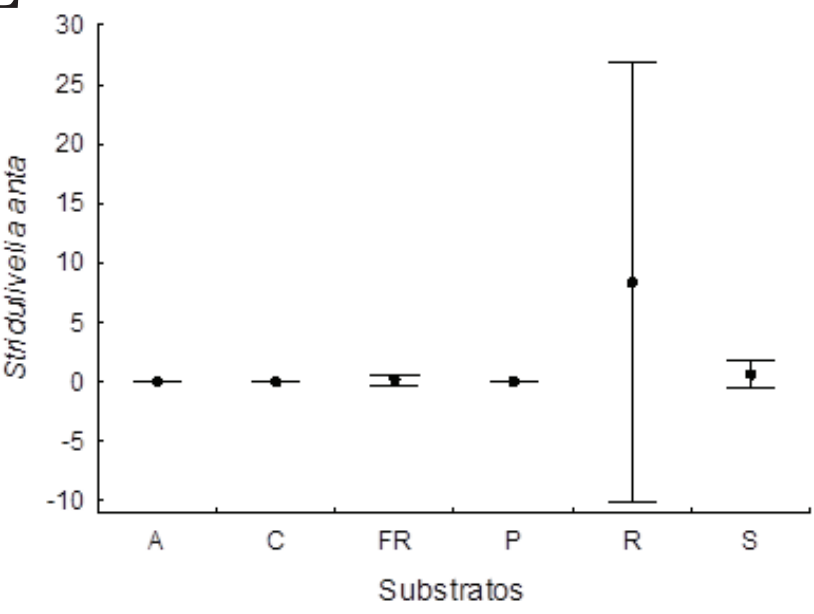

Figura 4. Abundância das morfoespécies de Gerromorpha nos substratos dos Córregos Santo Antônio e da Colher, Nova Xavantina, MT (2004/2005). A) Brachymetra sp.1; B) Stridulivelia anta; C) Neogerris lubricus; D) Rhagovelia elegans; F) Limnogonus aduncus aduncus [A, areia; C, cascalho; FC, folhiço de corredeira; FR, folhiço de remanso; P, pedra; R, raiz e S, superfície].

\section{DISCUSSÃO}

Padrão geral de distribuição da comunidade de Heteroptera. A maior abundância de Gerromorpha pode ser explicada pelo modo de vida gregário das duas principais famílias (Gerridae com cinco espécies/morfoespécies e Veliidae com seis espécies/morfoespécies) (SchuH \& Slater 1995), que propicia a coleta de muitos indivíduos em uma única amostra. Essas duas famílias podem constituir os grupos predadores de maior riqueza específica das comunidades límnicas (MоттA \& UiEDA 2004). Gerridae é a família de heterópteros com maior variedade de gêneros, tendo grande diversificação de formas de corpo correlacionadas à diversidade ecológica e adaptadas à patinação aquática, constituindo um grupo de habitat muito especializado (ANDERSEN 1982; Nieser \& MELo 1997). Veliidae são pequenos, de ocorrência em locais protegidos por mata ciliar e de pouco fluxo (Pelli et al. 2006), e geralmente em locais bem preservados (DiAs-Silva et al. 2010).

A alta abundância de Gerridae corrobora com os resultados encontrados na Bacia Hidrográfica do Rio Trombetas, onde estes contribuíram com 40,2\% dos indivíduos coletados (SAMPAIO \& PY- 
Daniel 1995) e a de Veliidae com dados de Dias-Silva et al. (2010), que trabalhando em córregos da mesma região obteve $44,5 \%$ dos Heteroptera coletados nessa família. De acordo com TAYLOR (1996), a densidade de cobertura de plantas é um dos fatores mais estudados na influência da distribuição dos semi-aquáticos. CABETTE et al. (2010), não registrou nenhum Gerromorpha em ambiente natural com forte abertura de dossel (Rio Darro, Bacia do Suiá-Miçú, MT). Assim, a abundância de Gerromorpha, com principal contribuição das famílias citadas, pode estar diretamente relacionada à presença de abrigos marginais e cobertura vegetal ofertada pelos Córregos da Colher e Santo Antônio, que apesar de algum impacto antrópico, ofereciam sombreamento na maioria dos ambientes.

Para Nepomorpha a maior abundância e maior número de morfoespécies foram representados pela família Naucoridae. Espécies dessa família apresentam uma alta capacidade de adaptação em diferentes substratos, conseguindo habitar águas correntes (Ribeiro et al. 1998), podendo também ocorrer em águas estagnadas aderidas a troncos, ramos, pedras ou até mesmo enterradas em solos arenosos, sendo considerada a família que possui os representantes mais adaptados a vida em ambientes lóticos (ROLdÁN-PÉREZ 1988; NiESER \& LOPEZ-RUF 2001). Apresentam uma grande diversidade de formas, que refletem em adaptações a uma grande variedade de nichos, o que poderia explicar a sua maior riqueza (MerRitT \& Cummins 1984).

Esses resultados podem ser explicados ainda por dois fatores: pela diversidade de habitats subaquáticos, onde vive a maioria dos Nepomorpha e poderia favorecer um maior particionamento de nicho, possibilitando a persistência de mais espécies, uma vez que Gerromorpha vive mais na superfície da lâmina da água; outro fator seria a perda de sombreamento em alguns locais de coleta em virtude da retirada da vegetação marginal, o que afetaria os indivíduos de Gerromorpha, que estariam mais susceptíveis as variações ambientais decorrentes desta ação, corroborando DiAsSilva et al. (2010) que obteve relações positivas de riqueza de Gerromorpha com integridade de hábitat, indicando que esses foram mais sensíveis a alterações na estrutura física dos rios que Nepomorpha.

Substratos. Nos substratos, a infraordem Gerromorpha apresentou maior riqueza na superfície da água, corroborando a hipótese proposta, uma vez que Gerromorpha é semi-aquático e tem modo de vida gregário, sendo encontrado sobre a coluna d'água (conforme exposto no parágrafo anterior), patinando ou escondido nas vegetações marginais, sendo fáceis de visualizar e, portanto fáceis de coletar. Segundo Molano-REdón et al. (2008) são altamente especializados para este tipo de habitat.

A maior riqueza de Nepomorpha se deu em substratos orgânicos, especialmente raízes, a abundância e riqueza elevadas encontradas nesse substrato podem estar relacionadas ao fato de propiciarem abrigo para várias espécies evitando que estes sejam carregados pela correnteza, já que adaptações para esta finalidade são comuns a diversos grupos (FIDELIs et al. 2008), bem como, a disponibilidade de presas, já que os substratos vegetais comportam maior número de espécies, porque este por si só, servem de recurso alimentar a grupos herbívoros e como local de oviposição para Heteroptera (KIKUCHI \& UIEDA 2005). Os tipos de substratos mais complexos fisicamente (folhas, cascalho ou pedras, macrófitas, musgo e madeira) geralmente suportam mais táxons que substratos estruturalmente mais simples (areia, e rochas) (Robson \& BARMuta 1998). Segundo Marques et al. (1999) a qualidade do hábitat constitui um dos fatores mais importantes para o sucesso da colonização e do estabelecimento dos macroinvertebrados em ambientes lênticos ou lóticos.

A alta riqueza na superfície apresentada por Nepomorpha pode estar relacionada a baixa profundidade apresentada no período de coleta e estes organismos terem sido capturados randomicamente. Outra hipótese seria a presença de adaptações para obter oxigênio na interface aquático/terrestre, como por exemplo, os adultos das famílias Naucoridae, Notonectidae, Corixidae e Nepidae que após capturar o oxigênio conseguem mantê-lo em seus reservatórios (NIESER \& MELO 1997).

Preferência por substrato das espécies mais abundantes de Heteroptera. Entre os Gerromorpha, as espécies se associaram a S, o que era esperado, Brachymetra sp.1, $N$. lubricus e L. a. aduncus (Gerridae) e R. elegans (Veliidae). Os Gerridae são habitantes comuns das superfícies das lagoas, lagos e áreas lóticas de córregos e rios, onde freqüentemente ocorrem em grandes agregações e são altamente especializados quanto ao seu habitat sobre a película da água (MOLANO-REDón et al. 2008); enquanto que representantes de Rhagovelia vivem sobre a água em locais com pequenas correntezas; a exceção de se deu com $S$. anta, que apresentou preferência por raiz, e isso se deve ao seu hábito de vida nas margens dos córregos entre as plantas marginais ou em remansos com muita vegetação (ROLDÁN-PÉREZ 1988; Nieser \& MELO 1997). Quanto à riqueza de Nepomorpha, a morfoespécie Ambrysus sp.1 mostrou associação ou preferência por FC, FR, R e S (Figura 2), que devem utilizar como abrigo e busca por presas, e o que indica que é capaz de obter recursos em uma ampla gama de microhábitats, sendo, portanto generalista.

Nossos resultados não corroboram os resultados de HerRmann et al. (1993), que estudaram a influência da velocidade da correnteza na escolha do substrato por Naucoridae, apontaram Ambrysus como o gênero mais comumente encontrado sob pedras em rios com correnteza. Sites \& Willig (1991), comentam que cascalho seria um dos substratos preferidos por muitas espécies desse gênero, estas incongruências percebidas mostram a necessidade das análises serem em nível de espécie, pois dentro de um mesmo gênero há variações importantes nos hábitos, expresso aqui pela morfoespécie Ambrysus sp.1.

Ainda desta infraordem, $M$. chinai apresentou preferência por colonizar a superfície da água, estes vivem em riachos limpos e locais de pouca correnteza como lagos, lagoas, tanques e poços com pouca ou nenhuma vegetação (NIESER \& MELo 1997), talvez relacionada à estratégia de alimentação, já que GitTELMAN (1975) observou que a espécie Martarega hondurensis Bare apresenta clara preferência por presas que eram encontradas flutuando na superfície. Além disso, segundo Giller \& McNeill (1981) a família Notonectidae possui espécies muito similares ecologicamente, que sendo nadadores ativos, são encontrados em uma ampla variedade de habitats.

De acordo com Subramanian \& Sivaramakrishnan (2005), a diversidade e abundância dos táxons varia entre os habitats e tais informações devem ser levadas em conta para a compreensão de suas distribuições espaciais e no desenvolvimento de ferramentas para o biomonitoramento. Variações na composição das comunidades nos córregos são freqüentemente associadas com mudanças nos tipos de substratos disponíveis em cada riacho amostrado (ARMITAGE et al. 2001) e alterações nas bacias hidrográficas ou no curso do riacho devem alterar a estrutura de microhábitats disponíveis, modificando assim, as assembleias.

Embora as espécies apresentem uma ampla preferência e distribuição nos substratos, foram detectados padrões recorrentes de associação que possibilitam generalizações para as duas subordens. Para trabalhos que visem analisar a diversidade de espécies de um determinado local, faz-se necessário uma amostragem dos vários tipos de substratos presentes nesses locais, dando ênfase em coletas na superfície da água (S) para Gerromorpha e nos substratos orgânicos (especialmente R) para Nepomorpha. Possibilitando assim, uma amostragem mais representativa da diversidade contida no ambiente estudado, já que estes contemplaram $83 \%$ das espécies coletadas em Gerromorpha e 64\% das espécies coletadas em Nepomorpha, da riqueza de espécies coletadas, respectivamente. Em resumo, a obtenção de sete espécies com associação especifica a 
determinados tipos de substratos nos dá informações ecológicas úteis para o monitoramento ambiental nos córregos do leste mato-grossense.

\section{AGRADECIMENTOS}

Ao laboratório de Entomologia da Universidade do Estado de Mato Grosso-UNEMAT pelo apoio institucional e acadêmico, ao Lourivaldo Amâncio de Castro pelo suporte de campo, ao CNPQ e a CAPES pela concessão de bolsa a Dias-Silva, K. e Giehl, N. F. S. A equipe do laboratório de entomologia pela contribuição nas coletas de dados. Ao especialista Dr. Felipe F.F. Moreira UFRJ, Laboratório de Entomologia pela confirmação da identificação do material de Gerromorpha. Ao Dr. Amintas Nazaret Rossete (Núcleo de análise ambiental-NANA) pela confecção do mapa da área de estudo. Ao PROCAD (proc. $n^{\circ}$ 109/2007) e FIDIPEX/ UNEMAT pelo fomento da pesquisa. Aos revisores anônimos pelas sugestões que auxiliaram na melhoria do manuscrito.

\section{REFERÊNCIAS}

Andersen, N.M., 1982. The semiaquatic bugs (Hemiptera, Gerromorpha). Phylogeny, adaptations, biogeography, and classification. Scandinavian Science Press, Klampenborg, Entomonograph. 455p.

Armitage, P.D., K. Lattmann, N. Kneebone \& I. Harris, 2001. Bank profile and structure as determinants of macroinvertebrate assemblages-season changes and management. Regulated Rivers: Research \& Management, 17: 543-556.

Assis, J.C.F., A.L. Carvalho \& J.L. Nessimian, 2004. Composição e preferência por microhabitat de imaturos de Odonata (Insecta) em um trecho de baixada do rio Ubatiba. MaricáRJ, Brasil. Revista Brasileira de Entomologia, 48: 273-282.

Burnham, K.P. \& W.S. Overton, 1978. Estimation of the size of a closed population when capture probabilities vary among animals. Biometrika, 65: 625-633.

Buss, D.F., D.F. Baptista \& J.L. Nessimian, 2003. Bases conceituais para a aplicação de biomonitoramento em programas de avaliação da qualidade da água de rios. Caderno de Saúde Pública, 19: 465-473.

Cabette, H.S.R., N.F.S. Giehl, K. Dias-Silva, L. Juen \& J.D. Batista, 2010. Distribuição de Nepomorpha e Gerromorpha (Insecta: Heteroptera) da Bacia do Rio Suiá-Miçú, MT: riqueza relacionada à qualidade de água e de hábitat, p. 113-137. In: Santos, J.E., C. Galbiati \& L.E. Moschini (Orgs.). Gestão e Educação Ambiental: Água, Biodiversidade e Cultura. São Carlos, RiMa, 426p.

Callisto, M., P. Moreno, M. Goulart, A. Medeiros, M. Petrucio, M. Moretti, N. Mayrink \& C.A. Rosa, 2002. The assessment of aquatic biodiversity along an altitudinal gradient at the Serra do Cipó (South-Eastern Brazil). Verhandlungen des Internationalen Verein Limnologie, 28: 1-4.

Colwell, R.K., 2005. EstimateS: Statistical estimation of species richness and shared species from samples. Version 7.5. [6.ob1].

Dias-Silva, K., H.S.R. Cabette, L. Juen \& Jr.P. De Marco, 2010. Habitat integrity and physical-chemical water variables influence on the structure of aquatic and semi-aquatic Heteroptera. Revista Brasileira de Zoologia, 27: 918-930.

Dufrêne, M. \& P. Legendre, 1997. Species assemblages and indicator species: the need for a flexible asymmetrical approach. Ecological Monographs, 67: 345-366.

Fidelis, L., J.L Nessimian \& N. Hamada, 2008. Distribuição espacial de insetos aquáticos em igarapés de pequena ordem na Amazônia Central. Acta Amazônica, 38: 127-134.

Galdean, N., M. Callisto, F.A.R. Barbosa \& L.A. Rocha, 2000. Lotic ecosystems of Serra do Cipó, Southeast Brazil: water quality and a tentative classification based on the benthic macroinvertebrate community. Journal of Aquatic Ecosystem Health \& Restoration, 3: 545-552.

Gittelman, S.H., 1975. The Ecology of Some Costa Rican Backswimmers (Hemiptera: Notonectidae). Annals of the
Entomological Society of America, 68: 511-518.

Giller, P.S. \& S. Mcneill, 1981. Predation strategies, resource partitioning and habitat selection in Notonecta (Hemiptera/ Heteroptera). Journal Animal Ecology, 50: 789-808.

Gotelli, N.J. \& A.M. Ellison, 2011. Princípios de estatísticas em ecologia. Artmed, 528p.

Goodwyn, P.J.P., 2001. Anew Hydrometra species from Argentina (Heteroptera: Hydrometridae). Florida Entomologist, 84: 127-130.

Herrmann, D.P., R.W. Sites \& M.R. Willig, 1993. Influence of current velocity on substratum selection by Naucoridae (Hemiptera) - an experimental approach via stream simulation. Environmental Entomology, 22: 571-576.

Heckman, C.W., 2011. Encyclopedia of South American Aquatic Insects: Hemiptera-Heteroptera Illustrated Keys to Known Families, Genera, and Species in South America. Springer, $679 \mathrm{p}$.

Hungerford, H.B., 1948. The Corixidae of the Western Hemisphere (Hemiptera). University of Kansas Science Bulletin, 32:1827.

Hynes, H.B.N., 1970. The ecology of stream insects. Annual Review of Entomology, 15: 25-42.

Kikuchi, R.M. \& V.S. Uieda, 2005. Composição e distribuição dos macroinvertebrados em diferentes substratos de fundo de um riacho no Município de Itatinga, São Paulo, Brasil. Entomologia y Vectores, 12: 1-18.

Marques, M.G.S.M., R.L. Ferreira \& F.A.R. Barbosa, 1999. A comunidadedemacroinvertebradosaquáticosecaracterísticas limnológicas das lagoas Carioca e da Barra, Parque Estadual do Rio Doce, MG. Revista Brasileira de Biologia, 59: 203210.

Mato Grosso, 1998. Zoneamento socioeconômico e ecológico de MT (Mapa Aoo2: Compartimentação climatológica). SEPLAN/MT, Disponível em: < www.seplan.mt.gov.br>.

Menke, A.S., 1979. The semiaquatic and aquatic Hemiptera of California. Bulletin of the California Insect Survey 21:1-166 $+x i$.

Merritt, R.W. \& K.W. Cummins, 1984. An introduction to the aquatic insects of North America. Dubuque, Kendall/Hunt Publishing Company, 311p.

Molano-Redón, F., I.T. Morales-Castaño \& C. Serrato-Hurtado, 2008. Clasificación y hábitats de Gerridae (Heteroptera Gerromorpha) en Colombia. Acta Biologica Colombiana, 13: 41-60.

Motta, R.L. \& V.S. Uieda, 2004. Diet and trophic groups of an aquatic insect community in a tropical stream. Brazilian Journal of Biology, 64: 809-817.

Neri, D.B., C.B. Kotzian \& A.E. Siegloch, 2005. Composição de Heteroptera aquáticos e semi-aquáticos na área de abrangência da U.H.E Dona Francisca, RS, Brasil: fase de pré- enchimento. Iheringia, Série Zoologica, 95: 421-429.

Nieser, N. \& M. Lopez-Ruf, 2001. A review of Limnocoris Stål (Heteroptera Naucoridae) in Southern South America east of the Andes. Tijdschrift Voor Entomologie, 144: 261-328.

Nieser, N. \& A.L. Melo, 1997. Os heterópteros aquáticos de Minas Gerais: guia introdutório com chave de identificação para as espécies de Nepomorpha e Gerromorpha. Belo Horizonte, Ed. UFMG, 18op.

Nieser, N., A. Pelli \& A.L. Melo, 1999. Two new Ambrysinae (Heteroptera: Naucoridae) from Minas Gerais, Brazil. Acta Societatis Zoologicae Bohemicae, 63: 157-163.

Pelli, A., N. Nieser \& A.L. Melo, 2006. Nepomorpha and Gerromorpha (Insecta: Heteroptera) from the Serra da Canastra, southwestern Minas Gerais state, Brazil. Lundiana, 7: 67-72.

Petts, G.E., 1994. Rivers: dynamic components of catchment ecosystems, p. 3-32. In: Calow, P. \& G.E Petts (Eds.). The River Handbook. Oxford, Blackwell Scientific, 536 p.

Ribeiro, J.R.I., J.L. Nessimian \& E.C. Mendonça, 1998. Aspectos da distribuição dos Nepomorpha (Hemiptera: Heteroptera) em corpos d'água na Restinga de Maricá, Estado do Rio de 
Janeiro, cap. 5, p. 113-128. In: Nessimian, J.L. \& A.L. Carvalho (Eds.). Ecologia de insetos aquáticos. Oecologia Brasiliensis, $309 \mathrm{p}$.

Robson, B.J. \& L.A. Barmuta, 1998. The effect of two scales of habitat architecture on benthic grazing in a river. Freshwater Biology, 39: 207-220.

Roldán-Pérez, G., 1988. Guía para el estudio de los macroinvertebrados acuáticos del departamento deAntioquia. Bogotá, Universidad de Antioquia, 217p.

Sampaio, R.T.M. \& V. Py-Daniel, 1995. A subfamilia Gerrinae (Hemiptera: Heteroptera: Gerridae) na Bacia Hidrográfica do rio Trombetas, Pará, Brasil. Acta Amazonica, 23: 83-94.

Schuh, R.T. \& J.A. Slater, 1995. True bugs of the world (Hemiptera: Heteroptera). Classification and natural history. Cornell University Press, Cornell, 336p.

Sites, R.W. \& M.R. Willig, 1991. Microhabitat associations of 3 sympatric species of Naucoridae (Insecta, Hemiptera). Environmental Entomology, 20: 127-134.
Subramanian, K.A. \& K.G. Sivaramakrishnan, 2005. Habitat and microhabitat distribution of stream insect communities of the Western Ghats. Current Science, 89: 976-987.

Taylor, S.J., 1996. Habitat preferences, species assemblages, and resource partitioning by Gerromorpha (Insecta: Heteroptera) in southern Illinois, with a faunal list and keys to species of the state. USA, Southern Illinois University, 690p.

Wise, D.H. \& M.C. Jr. Molles, 1979. Colonization of artificial substrates by stream insects: influence of substrate size and diversity. Hydrobiologia, 65: 69-74.

\section{Recebido em: 14/12/2012}

Aceito em: 14/o3/2013

\section{Como citar este artigo:}

Dias-Silva, K., H.S.R. Cabette, N.F.S. Giehl \& L. Juen, 2013. Distribuição de Heteroptera Aquáticos (Insecta) em Diferentes Tipos de Substratos de Córregos do Cerrado Matogrossense. EntomoBrasilis, 6(2): 132-140.

Acessível em: http://www.periodico.ebras.bio.br/ojs/index.php/ebras/article/view/302. doi:10.12741/ebrasilis.v6i2.302

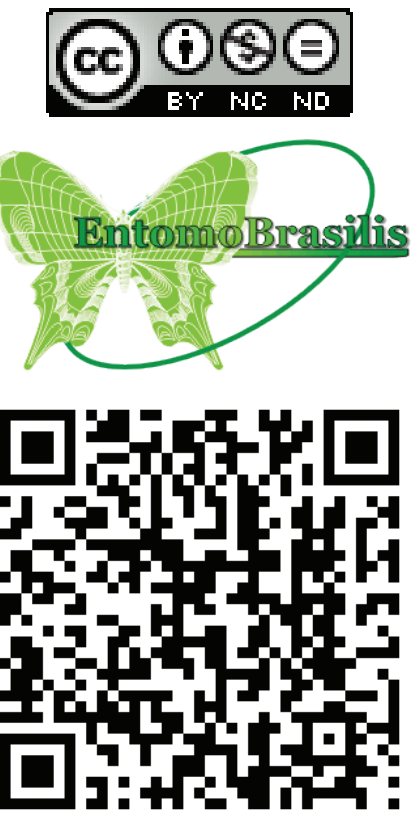

